\title{
A Semiempirical Approach to the Extension of Stress Cracking in Polymeric Materials under Creep Conditions
}

\author{
Takanori SArTo \\ Central Research Laboratory, Showa Denko Co., 2-24-60, \\ Ohta-ku, Tokyo 146, Japan.
}

(Received March 29, 1978)

\begin{abstract}
The aim of this work is to derive a semiempirical equation for the crackextension rate of stressed polymers. This is achieved by using the Dugdale model and assuming that an imaginary crack jerks and transfigures itself into a true crack when a plastic strain near the crack-tip reaches a critical value. The overall rate of plastic deformation is shown to play an important role in determining the rate of steady crack-extension. Finally, the life time is written as a function of the distribution of dislocation density, applied stress, the Newton viscosity, temperature, the stress sensitivity (or the activation volume) and some material constants. This formulation of the life time can be reduced to the so-called the Holland-Turner's equation and to the Zhurkov-Narsulaev's equation for the region of lowand high-stress applied, respectively.

KEY WORDS Stress Cracking / Crack-Extension Rate / Life Time / Dugdale Model / Plastic Zone / Overall Strain-Rate / Plastic Strain / Holland-Turner's Equation / Zhurkov-Narsulaev's Equation /
\end{abstract}

The mechanism of the stress cracking with and without an environmental agent is a matter of primary concern in practical usages of plastics for pipelines, cable sheaths and bottles and so on. Environmental stress cracking (ESC) has not been thoroughly solved yet to date. ${ }^{1}$ Fracture mechanics show empirically that the rate of crackextension $i$ relates to the stress intensity factor $K$ as follows. ${ }^{2-6}$

$$
\dot{l}=h K^{2 m}
$$

where, $m$ and $h$ are material constants affected by environments. The empirical eq 1 is powerful for life-time prediction and analysis of the fracture phenomena. However eq 1 does not necessarily indicate a clear mechanism of the growth of stress cracking, nor does it show the effect of material parameters, for example, molecular weight and yielding stress etc. on life-time. For a molecular design of high performance materials, it is desirable that any theory on crack-growth involves material parameters explicitly. Some approaches to this analysis were offered by several authors with various criteria and assumptions. For instances, Williams $^{7}$ or Knauss $^{8}$ investigated the rate of crack-growth in viscoelastic materials by the analysis of stress distribution at the crack-tip. On the other hand, the variation of the strain distribution in plastic zone at crack-tip was shown for three plastics by Ferguson, et al. ${ }^{9}$ Those works suggest that an extension of crack can be analyzed in terms of a distribution of plastic strain with proper assumption of a stress distribution at cracktip.

Successful applications of the Dugdale model $(D M)^{10}$ to the fracture of stressed polymers has been reported. ${ }^{11-14} \mathrm{DM}$ is based on the scheme in which a crack is preceded and bordered by the plastic zone with the traction being equal to the yield stress. In fact, one can readily observe the localized plastic deformations ahead of the cracktip by an optical or an electron microscopy. ${ }^{15}$ One of the plastic deformations (PD) in polymers is the craze which is a highly oriented and void structure noted above in all glassy polymers, ${ }^{16-18}$ and also in crystalline polymers. ${ }^{17,19}$ Other forms of PD are, for example, oblique markings called kink bands ${ }^{20-22}$ and the formation of fibers in oriented crystalline polymers. ${ }^{23}$ A plastically deformed zone ahead of the crack-tip can be mod- 
elled by a continuous array of edge dislocations ${ }^{24}$ with time dependent DM after Riedel. ${ }^{25}$ In this sense, a distribution of plastic strain is a distribution of the dislocation density, and which is given by Rice $^{26}$ as follows.

$$
\begin{gathered}
y(x, t)=y(, 0 t)\left\{\xi-\left(\frac{x}{2 r}\right) \ln \left(\frac{1+\xi}{1-\xi}\right)\right\}, \\
\text { with } \xi=\left(1-\frac{x}{r}\right)^{1 / 2}
\end{gathered}
$$

where, $y(x, t)$ is the half thickness of plastic zone, or plastic strain parallel to traction, at the time $t$ and the distance $x$ from a crack-tip, $y(0, t)$ is one half of the crack opening displacement (COD), $r(t)$ is the length of plastic zone at time $t$. We think here that the distribution of plastic strain moves with the crack tip in the course of time, and satisfies eq 2 at any time. The COD and $r(t)$ can be written by DM with the half crack-length $l(t)$ as $^{13}$

$$
\begin{gathered}
\mathrm{COD}=2 y(0, t)=\left(\frac{8}{\pi}\right) \varepsilon_{\mathrm{Y}} l(t) \ln \left[1+\frac{r(t)}{l(t)}\right] \\
r(t)=\left\{\sec \left(\frac{\pi \sigma_{\mathrm{a}}}{2 \sigma_{\mathrm{Y}}}\right)-1\right\} \zeta l(t) \approx\left(\frac{\zeta}{8}\right)\left(\frac{\pi \sigma_{\mathrm{a}}}{\sigma_{\mathrm{Y}}}\right)^{f} l(t), \\
\text { with } f \approx 2
\end{gathered}
$$

where, $\zeta$ is unity for DM, and

$$
\zeta=\left(\frac{\varepsilon_{\mathrm{Y}}}{\varepsilon_{\mathrm{c}}}\right) \phi(t)
$$

for the Kitagawa-Motomura model ${ }^{27}$ that takes creep behaviour into account with a creep compliance $\psi(t)$. In the above equations, $\varepsilon_{\mathrm{c}}, \varepsilon_{\mathrm{Y}}$ and $\sigma_{\mathrm{Y}}$ are the craze-initiation critical strain, the yield strain and the yield stress, respectively. The experimental results are well explained by these equations of $\mathrm{DM}$ when the applied stress $\sigma_{\mathrm{a}}$ in eq 4 is replaced by the corrected stress $\sqrt{\pi} \sigma(t)$ as

$$
\sigma_{\mathrm{a}}(t)=\sigma(t)=\sigma_{0} \Theta(t) \frac{Y}{\sqrt{\pi}}
$$

where $Y$ is the finite width correction factor, ${ }^{28} \sigma_{0}$ is the initial applied stress and $\Theta(t)$ is the time function of the stress relaxation in the case of testing under constant strain.

On the basis of this information, we intend to derive an equation for the crack-extension rate in stressed polymers by means of a simple geometri- cal model as a criterion of crack-growth, and moreover to analyze and predict the factors affecting the life time. For this purpose, we treat some flaws as inevitably preexistent in real materials, and develope an argument in terms of steady crackextension under creep conditions without mentioning how an initial crack is generated. (Symbols in this article are listed in the appendix.)

\section{EXPERIMENTAL AND RESULTS}

The test of the environmental stress-cracking resistivity (ESCR) of high density polyethylenes (HDPE) was carried out by the bent-strip method ${ }^{29}$ at $50^{\circ} \mathrm{C}$ in a $10-\%$ aqueous solution of Nonion $\mathrm{NS} 210^{\circledR}, \mathrm{C}_{9} \mathrm{H}_{19}-\ll-\mathrm{O}\left(\mathrm{CH}_{2} \mathrm{CH}_{2} \mathrm{O}\right)_{n} \mathrm{H}$, i.e., poly(oxyethylene) nonylphenyl ether by Nippon Oil \& Fats Co. Ltd. One can usually see a crack when half the crack-length reaches $c a .0 .02 \mathrm{~cm}\left(=l_{1}\right)$. The results on the life time of $F_{50}$ in hour and some

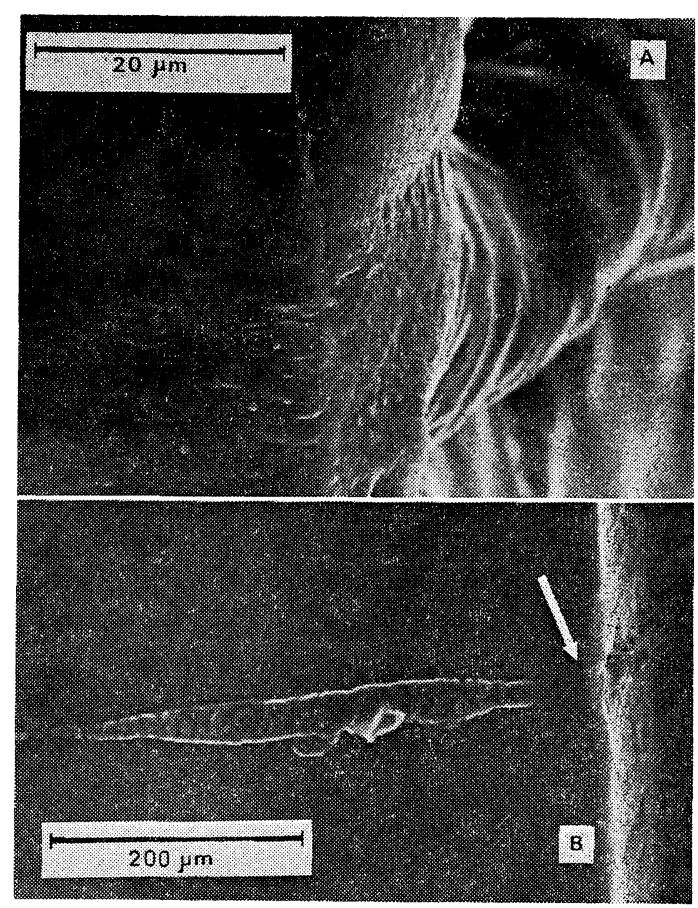

Figure 1. Plastic zone profile of a bent strip after ESCR test (B), and its enlargement of the arrow-tip region (A) where microcracks are observed here and there. This sample has code 3 (refer to the table inserted in Figure 5) The direction of notch and bending stress are vertical. 

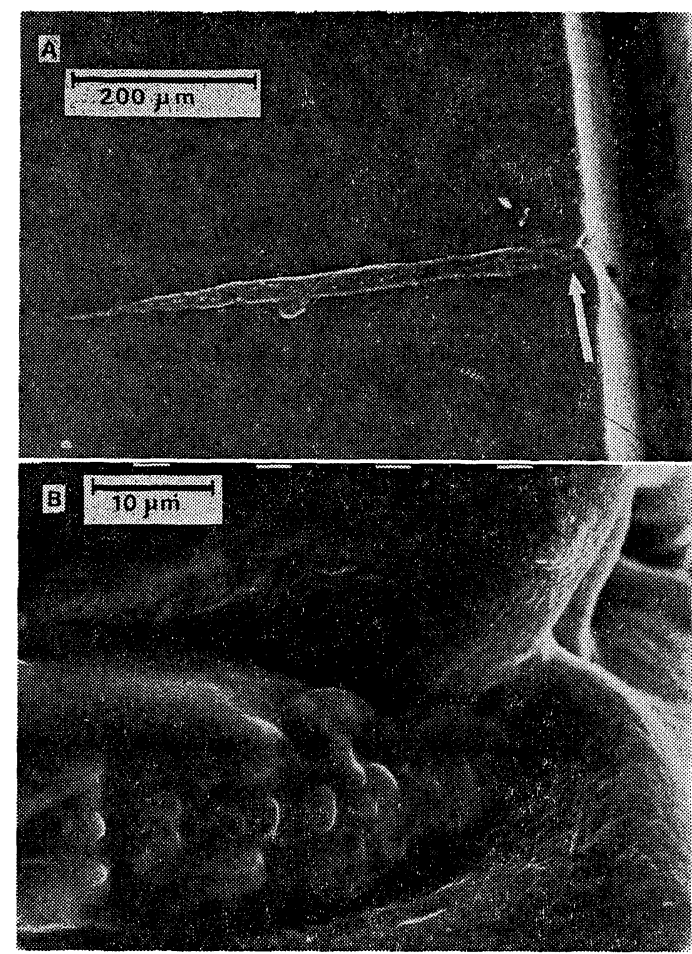

Figure 2. Plastic zone profile of a bent strip after ESCR test (A), and its enlargement of arrow-tip region (B) where the initiation of a crack is observed. The direction of notch and bending stress are vertical. Sample code, 2B.

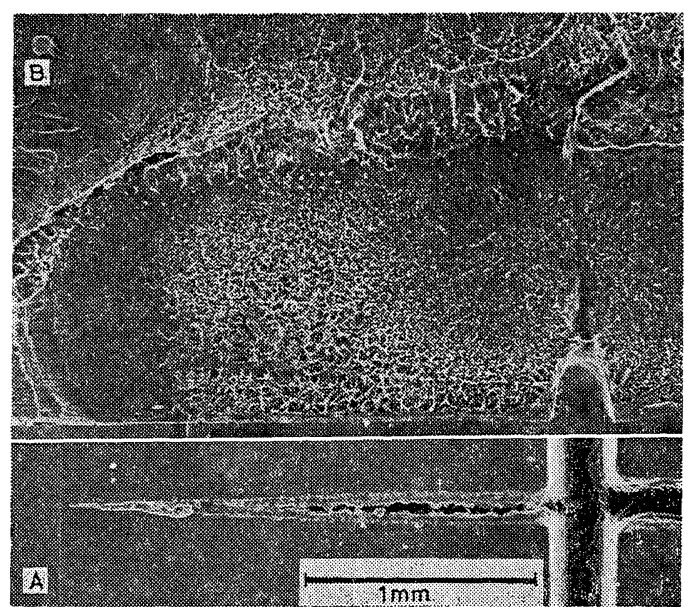

Figure 3. Crack and plastic zone profile of a bent strip after ESCR test (A) and its clove surface at the temperature of liquid nitrogen cooling (B) where cleavage facets and glide planes in the initiationregion, and elongated dimples in the propagationregion of ductile rupture are observed. Sample code, B.

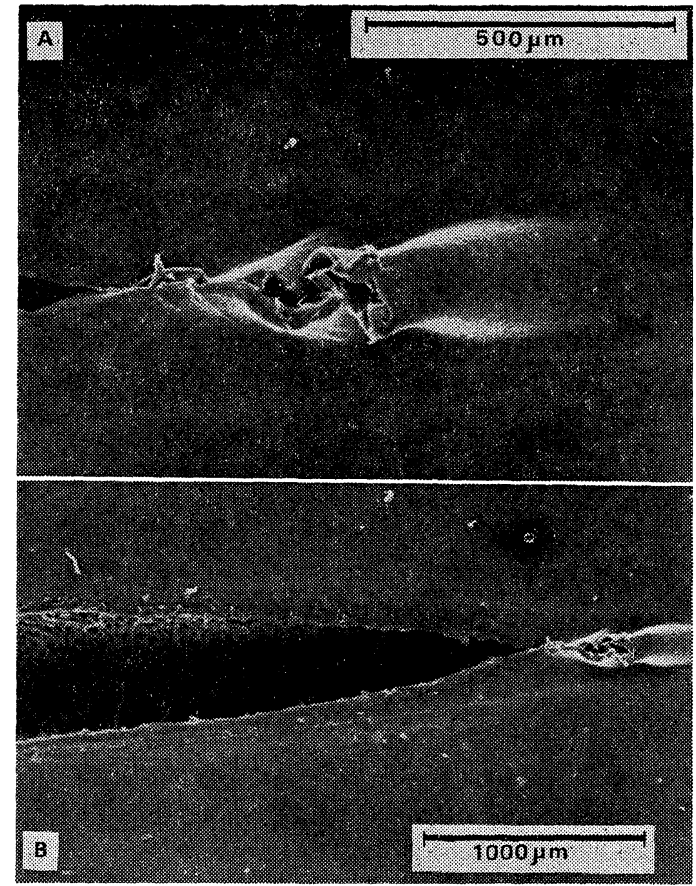

Figure 4. Crack and plastic zone (B) and its enlargement of crack-tip (A) where preceded and bordered zone of plastic deformation can be clearly observed. Sample code, 2 E.

characteristics are listed in the table inserted in Figure 5. The yield strength and strain of HDPE used here are $c a .250-280 \mathrm{~kg} / \mathrm{cm}^{2}$ and $c a .13$ $16 \%$, respectively at the tensile speed of $50 \mathrm{~mm} /$ min.

The profile of ESC and the fractured surface of HDPE were observed by the scanning electron microscope (SEM) of Model JSM-T20 by JEOL Ltd. The fractographs are shown in Figures 1-4. The plastic zone (PZ) followed by many microcracks and the starting of a macrocrack could be observed respectively in Figures 1 and 2, although the macrocracks grown were not observed in both figures. Figure $3 \mathrm{~A}$ is a profile of preceded $\mathrm{PZ}$ and a crack extended at right angles with the notch of the bent strip, and Figure $3 B$ is a fractography of the crack shown in Figure $3 \mathrm{~A}$ clove in two at the temperature of liquid nitrogen cooling. $\mathrm{PZ}$ at crack-tip and crack-border can be observed clearly in Figure 4, whereby we can envisage a model of crack-extension as shown in Figure 7.

The length of PZ, $r$ and the half crack-length, $l$ were obtained from measuring ESC profiles with 


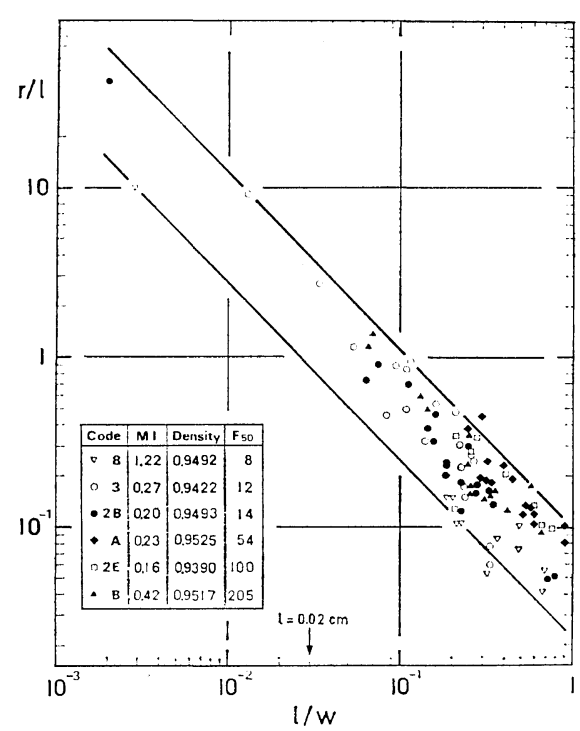

Figure 5. The ratio of plastic-zone length $(r)$ to crack length $(l) v s . l / w$ after the bent-strip test of ESCR, ${ }^{2 \theta}$ where $w$ is the half width of the strip being equal to $0.635 \mathrm{~cm}$. The unit of $F_{50}$ is in hour.

the aid of SEM observation. The plot of $r / l v s$. $l / w(w=$ half width of the strip $=0.635 \mathrm{~cm})$ is shown in Figure 5 which indicates that $r / l$ decreases with increasing $l / w$, and $r$ is approximately proportional to $l^{1-\hat{o}}$ with a positive constant, $\delta=-\mathrm{d} \ln (r / l) /$ $\mathrm{d} \ln (l / w)$, less than or equal to $c a$. unity.

The relaxation of the bending stress of the bent strip unnotched in air and in Nonion NS210 ${ }^{\circledR}$ was carried out at $20^{\circ} \mathrm{C}$ by a tensile tester (Tensilon UTM-III-100 by Toyo Baldwin Co. Ltd.) with compression untill the gap was equal to the width of the bent-strip holder $(1.20 \mathrm{~cm})$. After the initial region of the relaxation stress was continuously measured under the fixed gap, the strip was set in the sample holder by sliding it from the gap of the tester to expose it to the environment at $20^{\circ} \mathrm{C}$ or $50^{\circ} \mathrm{C}$. In the subsequent measurement of stress, the bent strip was reset in the tester by sliding it gently from the holder; these operations were repeated with one strip at $20^{\circ} \mathrm{C}$ and various strips at $50^{\circ} \mathrm{C}$. The stress relaxation curve is depicted in Figure 6 which shows that $\sigma(t) / \sigma_{0}$ decreases with time and approaches asymptotically at about $0.2-$ 0.4 within $c a .2 \mathrm{~h}$ in air at $20^{\circ} \mathrm{C}$, or in a very short time at $50^{\circ} \mathrm{C}$ compared with the life time of the ESCR. The value of $\sigma\left(t_{\infty}\right) / \sigma_{0}$ at $50^{\circ} \mathrm{C}$ with and without liquid environment is lower than that at $20^{\circ} \mathrm{C}$ in the air. This fact indicates that the internal stress is apt to relax drastically with an increase in temperature. This is justified by the definition of the internal stress as is the stress at $[\mathrm{d} \sigma(t) / \mathrm{d} t]=0$ in the stress relaxation. ${ }^{49}$

Those experimental results will be used in order to simplify the mathematical treatments of this work.

\section{THEORETICAL}

\section{A Model of Crack-Extension}

A model of crack-extension is shown in Figure 7

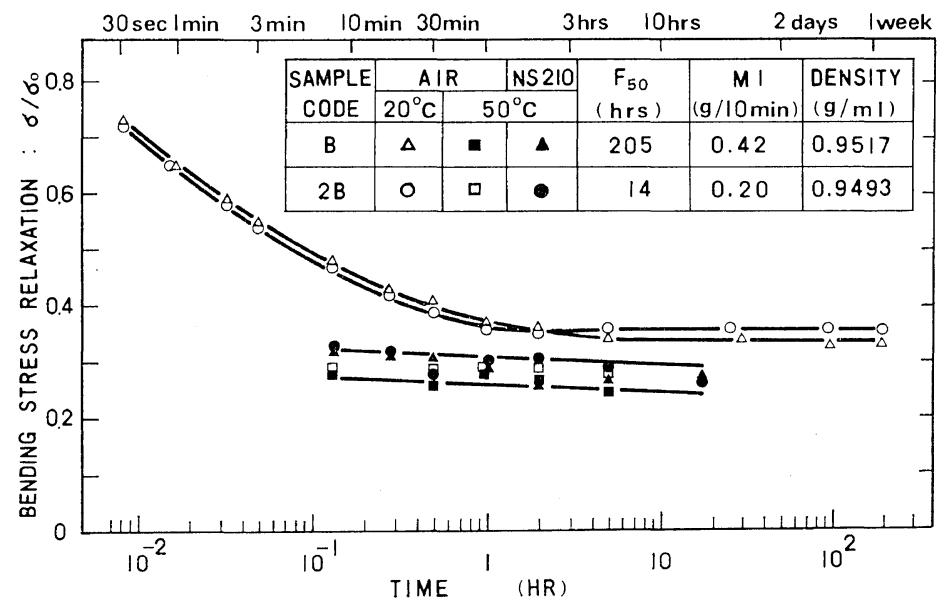

Figure 6. The stress relaxation curve of the bent strips unnotched, where initial bending stress $\sigma_{0}$ is $. c a .15 \mathrm{~kg} / \mathrm{cm}^{2}$. 


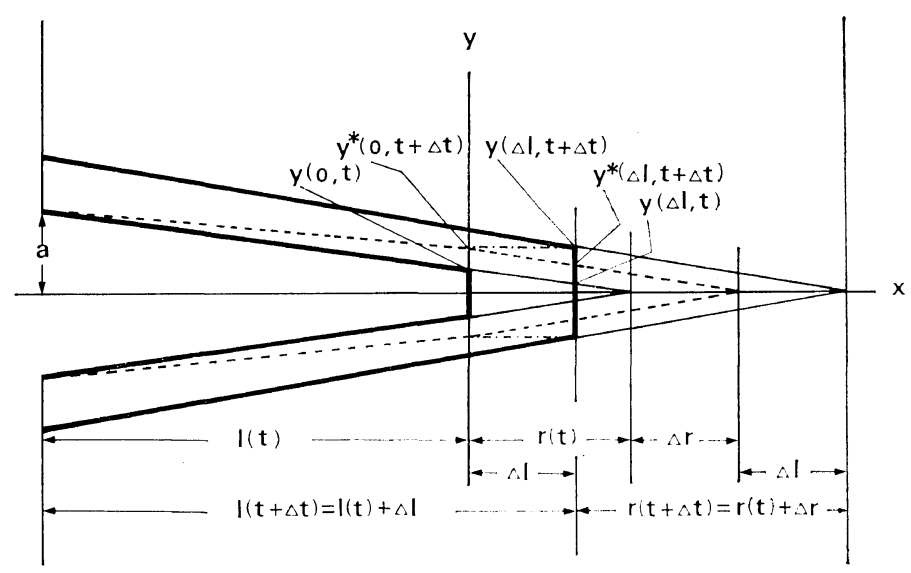

Figure 7. A modified Dugdale model of a crack-extension with an imaginary crack (dashed line) to jerk along the dash-dot-dashed line. The thick solid line is the border of a crack.

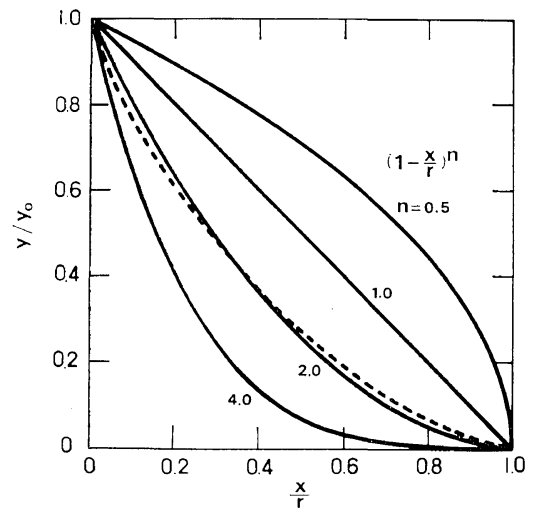

Figure 8. Distribution curves of plastic strain $\left(y / y_{0}\right.$ with $y_{0}$ as a COD) along plastic zone ( $x / r$ with $x$ as a distance from crack-tip), where solid line is eq 7 and dashed line is eq 2 proposed by Rice. ${ }^{26}$

and assumes that the plastic zone (PZ) preexists ahead of the crack. We propose eq 7 instead of eq 2 for the strain distribution of PZ, or a distribution of the dislocation density after Riedel $^{25}$; $y(x, t)=y(0, t) \xi^{2 n}=y(0, t)\left(1-\frac{x}{r}\right)^{n} \quad$ with $n>0$.

Equation 7 is simpler, and is a close approximation to the complicated eq 2 , especially in the case of $n=2$, as shown in Figure 8. Equation 7 can also represent a generalized profile for the distribution of the dislocation density along PZ by varying the shape parameter $n$ of the distribution.

In the course of time, $r(t)$ changes to $r(t+\Delta t)$ as

$$
r(t+\Delta t)=r(t)+\frac{\mathrm{d} r}{\mathrm{~d} t} \Delta t=r(t)+\Delta r
$$

This change brings about a thickening of $\mathrm{PZ}$, $y^{*}(\Delta l, t+\Delta t)$ and growth of COD, $2 y^{*}(0, t+\Delta t)$ which should be respectively written using eq 7 and 3 as

$$
y^{*}(\Delta l, t+\Delta t)=y^{*}(0, t+\Delta t)\left\{1-\frac{\Delta l}{r(t+\Delta t)}\right\}^{n}
$$

and

$$
y^{*}(0, t+\Delta t)=\left(\frac{4 \varepsilon_{\mathrm{Y}}}{\pi}\right) l^{*}(t+\Delta t) \ln \left\{1+\frac{r(t+\Delta t)}{l *(t+\Delta t)}\right\}
$$

with

$$
l *(t+\Delta t)=l(t+\Delta t)=l(t)+\frac{\mathrm{d} l}{\mathrm{~d} t} \Delta t=l(t)+\Delta l
$$

where $l^{*}(t+\Delta t)$ is the length of an imaginary crack assumed to have already extended to $l(t+\Delta t)$. The introduction of $y^{*}(\Delta l, t+\Delta t)$ and $l^{*}(t+\Delta t)$ is intended for the implicit consideration of all kinds of time dependent plastic deformation induced by applied stress. Since the imaginary expansion of PZ and COD given by eq 9 and 10 might be unstable and transient, we have set up a model 
such that a crack and PZ jerks and proceeds a distance $\Delta l$ for a time $\Delta t$ at a critical increment of plastic strain in PZ.

A criterion for the crack-extension is thus formulated by a geometrical approximation of $y(\Delta l$, $t+\Delta t)=y^{*}(0, t+\Delta t)$, and by the constant strain of $\mathrm{PZ}$ as

$$
\begin{aligned}
& \frac{y(\Delta l, t+\Delta t)-y(\Delta l, t)}{y(\Delta l, t)} \\
& =\frac{y^{*}(0, t+\Delta t)-y(\Delta l, t)}{y(\Delta l, t)}=\Delta u
\end{aligned}
$$

where $\Delta u$ is the increment of overall plastic strain of $\mathrm{PZ}$, and can be rewritten as

$$
\Delta u=\dot{u} \Delta t
$$

with $\dot{u}$ as a constant for the overall strain rate of the plastic deformation related to, for instance, the product of the dislocation velocity, the Burger's vector and the dislocation density (the number of dislocations per unit volume). ${ }^{30}$ Later on, $\dot{u}$ will be discussed.

\section{Crack-Extension Velocity}

One obtains eq 14 from eq 12 and 7 ,

$$
1+\Delta u=\frac{y^{*}(0, t+\Delta t)}{y(\Delta l, t)}=\frac{y^{*}(0, t+\Delta t)}{y(0, t)}\left\{1-\frac{\Delta l}{r(t)}\right\}^{-n}
$$

and rearrangement of eq 14 with eq 3 and 10 as

$$
\begin{aligned}
1+\Delta u= & \frac{l(t+\Delta t) \ln \{1+[r(t+\Delta t) / l(t+\Delta t)]\}}{l(t) \ln \{1+[r(t) / l(t)]\}} \\
& \times\left\{1-\frac{\Delta l}{r(t)}\right\}^{-n}
\end{aligned}
$$

wherewith we can easily derive an equation for crack-extension velocity.

(i) For the small crack, $l(t) \leq l_{1}$

The lower limit of $l(t)$ measurable by the eye, $l_{1}$ is $c a .0 .02 \mathrm{~cm}$. The relation $(r / l) \geq 1$ is shown for the region $l \leq l_{1}$ in Figure 5. Then we may approximate as follows.

$$
\frac{\ln \{1+[r(t+\Delta t) / l(t+\Delta t)]\}}{\ln \{1+[r(t) / l(t)]\}} \approx 1
$$

and

$$
\frac{l(t+\Delta t)}{l(t)}=1+\frac{\Delta l}{l(t)}
$$

So eq 15 may be reformed as

$$
1+\Delta u=1+\dot{u} \Delta t=1+\left(\frac{n}{r}+\frac{1}{l}\right) \Delta l
$$

hence, we obtain

$$
\begin{aligned}
\frac{\mathrm{d} l}{\mathrm{~d} t} & =\frac{\dot{u}}{(n / r)+(1 / l)}=\frac{\dot{u} r(t)}{n+\{r(t) / l(t)\}} \\
& \approx \dot{u} l(t) \quad \text { for } \quad(r / l) \gg n \approx 2 .
\end{aligned}
$$

(ii) For the large crack, $l(t)>l_{1}$

Since the relation $(r / l)<1$ for $l(t)>l_{1}$ is given by Figure 5, we may approximate as

$$
\frac{\ln \{1+[r(t+\Delta t) / l(t+\Delta t)]\}}{\ln \{1+[r(t) / l(t)]\}} \approx \frac{r(t+\Delta t) l(t)}{l(t+\Delta t) r(t)}
$$

and reform eq 15 with eq 21 and 8 ,

$$
\begin{aligned}
& 1+\Delta u=1+\dot{u} \Delta t \approx \frac{r(t+\Delta t)}{r(t)}\left\{1+\frac{n \Delta l}{r(t)}\right\} \\
& \approx 1+\left\{\frac{n \Delta l+\Delta r}{r(t)}\right\}
\end{aligned}
$$

where $\Delta r$ is the increment of the $\mathrm{PZ}$ length, and given in terms of eq $4-6$ as

$$
\Delta r=\Phi /(t) Y^{2} \Delta l
$$

with

$$
\Phi=\frac{\pi}{8}\left(\frac{\varepsilon_{\mathrm{Y}}}{\varepsilon_{\mathrm{c}}}\right)\left(\frac{\sigma_{0}}{\sigma_{\mathrm{Y}}}\right)^{2}
$$

and

$$
\Lambda(t)=\Theta^{2}(t) \psi(t) .
$$

In this case, crack-extension velocity can be written as

$$
\begin{aligned}
\frac{\mathrm{d} l}{\mathrm{~d} t} & =\frac{\dot{u} r(t)}{n+\Phi \Lambda(t) Y^{2}}=\frac{\dot{u} \Phi \Lambda(t) Y^{2} l(t)}{n+\Phi \Lambda(t) Y^{2}} \\
& \approx \frac{\dot{u}}{n} r(t) \text { for } \Phi \Lambda(t) Y^{2} \ll n .
\end{aligned}
$$

The characteristic of eq 19, 20 and eq 26, 27 is that the crack-extension rate is proportional to the velocity of the plastic deformation, which is in agreement with the empirical result of Nicholson for stainless steel. ${ }^{31}$

\section{DISCUSSION}

Here, let's touch upon $\dot{u}, \Lambda(t)$ and $Y$ before de- 
scribing some applications of theory to practical problems. Assuming that plastic deformation process is a single rate process of thermal activation, overall strain rate $\dot{u}$ can be written as ${ }^{32}$

$$
\dot{u}=\dot{u}_{0} \exp (-Q / k T) \exp \left(B \sigma_{\mathrm{e}}\right)
$$

where, $Q, k, T, \sigma_{\mathrm{e}}$ and $B$ are the true activation energy, Boltzmann's constant, the absolute temperature, the effective stress, that is, $\sigma_{\mathrm{e}}=\sigma-\sigma_{i}$, with the applied stress $(\sigma)$ and the internal stress $\left(\sigma_{i}\right)$, and $B=b^{*} \theta$, respectively. The parameter $b^{*}$ relates to the stress sensitivity of plastic deformations, $s$, as follows ${ }^{33}$

$$
s=\left(\frac{\partial \ln \dot{u}}{\partial \sigma_{\mathrm{e}}}\right)_{\mathrm{T}, \mathrm{P}}=\frac{d \dot{u}}{\dot{u} d \sigma_{\mathrm{e}}}=b^{*}=\frac{v^{*}}{k T}
$$

where, $v^{*}$ is called the shear activation volume defined as

$$
v^{*}=-\left(\frac{\partial Q_{\mathrm{a}}}{\partial \sigma_{\mathrm{e}}}\right)_{\mathrm{T}},
$$

with $Q_{a}$ as the apparent activation energy. The parameter $\theta$, i.e., $\Theta\left(t_{\infty}\right)$ is equal to unity for the case of constant stress and $\theta=0.2-0.4$ for the case of constant strain as shown in Figure 6. The order of magnitude of $v^{*}$ for $\mathrm{HDPE}^{32}$ and other plastics ${ }^{34}$ is reported as $c a .1-10 \mathrm{~nm}^{3}$ which is 2 to 20 times the statistical segment-volume of polymers. ${ }^{34}$ Though the physical meaning of $v^{*}$ is not necessarily clarified, $v^{*}$ can be interpreted simply as the volume difference between the activated and the normal state of the mobile entity, ${ }^{35}$ or as the quantity related to the work needed to change the volume of the system due to the bond breaking and/or atomic rearrangement process. $^{36}$ So there is a possibility that environmental reagents affect the materials through $v^{*}$ or the stress sensitivity, $s$ as well as the activation energy, $Q$.

The overall strain-rate constant $\dot{u}_{0}$ is written in this paper as a summation of the term $\dot{\phi}^{*}(t)=\left(\mathrm{d} \phi^{*}\right)$ $\mathrm{d} t$ ) due to a creep compliance $\psi^{*}(t)$ and the term $\dot{\gamma}(t)$ arising from the compliance of other plastic deformations including the formation and growth of voids.

With

$$
\phi^{*}(t)=\frac{u(t)}{\sigma_{0}}=j_{0}+\frac{t}{\eta_{0}}+J(t)
$$

and the creep function $J(t)$, i.e.,

$$
J(t)=\left\{1-\exp \left(-\frac{t}{\tau^{\prime}}\right)\right\} j_{\infty}
$$

we have

$$
\dot{u}_{0}=\frac{\mathrm{d} \psi^{*}}{\mathrm{~d} t}+\dot{\gamma}(t)=\frac{1}{\eta_{0}}+\frac{j_{\infty}}{\tau^{\prime}} \exp \left(-\frac{t}{\tau^{\prime}}\right)+\dot{\gamma}(t)
$$

where, $u(t), \sigma_{0}, j_{0}, \eta_{0}$, and $\tau^{\prime}$ are the overall strain at the time $t$, the constant (or initial) applied stress, an instantaneous creep compliance, the Newton viscosity and a retardation time, respectively. The term $j_{\infty}$ is equal to $J(\infty)$, and the equillibrium creep compliance is given by the sum of $j_{0}$ and $j_{\infty}$.

Since the strain rate in steady state creep is constant as indicated by many experiments, we may introduce here the following replacement.

$$
\frac{j_{\infty}}{\tau^{\prime}} \exp \left(-\frac{t}{\tau^{\prime}}\right)+\dot{\gamma}(t) \approx \frac{\rho}{\eta_{0}}
$$

where $\rho$ is a constant independent on time. Hence, from eq 33 and 34 one has

$$
\dot{u}_{0} \approx \frac{1}{\eta_{0}}+\frac{\rho}{\eta_{0}}=\frac{G}{\eta_{0}} \quad \text { (in the dimension of Time }{ }^{-1} \text { ) }
$$

with $G=1+\rho=$ material constant at a given temperature, stress and environments. The elasticity of a material may be included by the parameter $G$, and $\dot{\phi}^{*}(t)$ is substantially incorpolated into $\dot{u}_{0}$ through $\eta_{0}$ and $G$.

The time function $A(t)=\Theta^{2}(t) \psi(t)$ given by the eq 25 depends strongly upon the test method because of the stress relaxation term $\Theta(t)=1$ for the test under constant stress, and the creep compliance $\phi(t)=1$ for constant strain. As shown in eq 33, the effect of creep was taken into account through $\dot{\phi}^{*}$. The creep compliance $\phi(t)$ is then written simply as the constant $\psi_{0}$ for the initial state of creep, and otherwise as a constant $\psi$. On the other hand, $\Theta(t)$ may be certainly written as $\exp (-t / \tau)$ with a relaxation time $\tau$ for the initial state of stress relaxation, and as nearly constant for a more advanced state as shown in Figure 6. The aspect of stress relaxation in Figure 6 is consistent with the published data of Kubát, et al. ${ }^{37}$ So $\Lambda(t)$ is given with $c=2 / \tau$ as

$$
\Lambda(t)=\psi_{0} \exp (-c t)
$$

at an early state of deformation, or for small $l(t)$, 
and as

$$
\Lambda(t)=\psi \theta^{2}=\lambda \text { with } \theta=\Theta\left(t_{\infty}\right)
$$

at later state, or for large $l(t)$.

Next, the finite width correction factor $Y$, recommended for use, is usually formulated as

$$
\begin{aligned}
Y=\sum_{i=0}^{4} Y_{i}\left(\frac{l}{w}\right)^{i}= & 1.99-0.41(l / w)+18.7(l / w)^{2} \\
& -38.48(l / w)^{3}+53.85(l / w)^{4}
\end{aligned}
$$

with $Y_{0} \approx 2$ for the single-edge-cracked plate in tension, and for the bending deformation. ${ }^{28}$ In the ideal case of a through linear crack, we can correct the effect of cross sectional change by the factor,

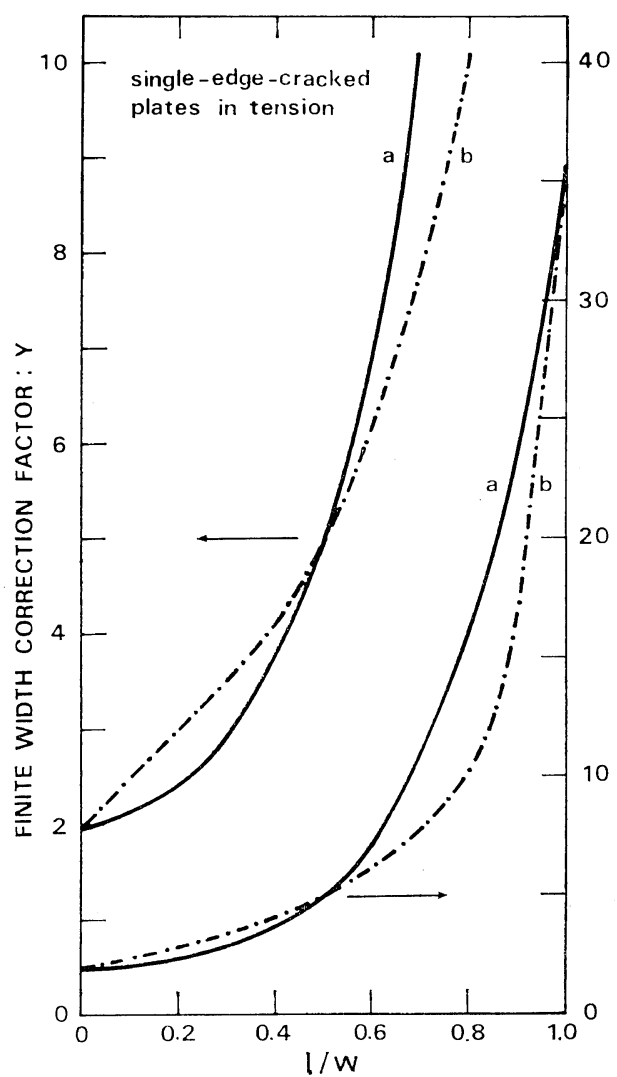

Figure 9. Finite width correction factor $(Y)$ vs. the ratio of crack length to half width of the strip $(l / w)$, where the solid line is eq 38 for the single-edge-cracked plate in tension proposed by Brown and Srawley, ${ }^{28}$ and the dash-dot-dashed line is eq 40 with $\alpha=2.107$, $\beta=1.059$ and $\nu=0.6462$.

$$
Y=w /(w-l)=1 /\{1-(l / w)\}
$$

Avoiding divergency of eq 39 when $l$ reaches half the width of a specimen $w$, a modified form of eq 39 , i.e.,

$$
Y=\alpha /\left\{\beta-(l / w)^{\nu}\right\} \text { with } \alpha, \beta \text { and } \nu>0
$$

is used for the real crack instead of eq 38 in this article. Though a closed form of eq 40 is not consistent enough with the power-series form of eq 38 as shown in Figure 9, both of these are nearly equal to $Y_{0}=\alpha / \beta \approx 2$ for $l / w \leq 0.1\left(c f ., l / w \approx 0.03\right.$ for $l=l_{1}$ $=0.02 \mathrm{~cm}$ ), and drastically increases in the region of $l / w \geqq 0.5$.

\section{Time Dependence of Crack-Length}

(i) For the small crack, $l(t) \leq l_{1}$

Rearranging eq 19 with eq $4-6$ and

$$
\Phi_{0}=\left(\frac{8}{\pi}\right)\left(\frac{\varepsilon_{\mathrm{Y}}}{\varepsilon_{\mathrm{c}}}\right)\left(\frac{\sigma_{0}}{\sigma_{\mathrm{Y}}}\right)^{2} Y_{0}^{2}=\Phi Y_{0}^{2},
$$

one has

$$
\frac{\mathrm{d} l}{\mathrm{~d} t}=\frac{\dot{u} \Phi_{0} \Lambda(t) l(t)}{n+\Phi_{0} \Lambda(t)} .
$$

Integrating eq 42 with $\Lambda(t)=$ variable as given in eq 36 and $Y \approx Y_{0}=$ constant, i. e.,

$$
\int_{l_{0}}^{l} \frac{\mathrm{d} l}{l}=\int_{0}^{t} \frac{\dot{u} \Phi_{0} \Lambda(t) \mathrm{d} t}{n+\Phi_{0} \Lambda(t)}=-\frac{\dot{u}}{c} \int_{\Lambda(0)}^{\Lambda(t)} \frac{\Phi_{0}}{n+\Phi_{0} \Lambda} \mathrm{d} \Lambda
$$

we have

$$
\ln \left(\begin{array}{c}
l \\
l_{0}
\end{array}\right)=\frac{\dot{u}}{c} \ln \left\{\frac{n+\phi_{0} \Phi_{0}}{n+\phi_{0} \Phi_{0} \exp (-c t)}\right\}
$$

or

$$
\exp (-c t)=\left(\frac{n}{\phi_{0} \Phi_{0}}+1\right)\left(\frac{l}{l_{0}}\right)^{-c / \dot{u}}-\frac{n}{\phi_{0} \Phi_{0}}
$$

then

$$
t=\ln \left\{\left(\frac{l_{0}}{l}\right)^{c / \dot{u}}-\frac{n}{\phi_{0} \Phi_{0}}\left[1-\left(\frac{l_{0}}{l}\right)^{c / \dot{u}}\right]\right\}^{-1 / c}
$$

or, for $t \ll 1 / c=\tau / 2$, from eq 45

$$
t \approx \frac{1}{c}\left(\frac{n}{\psi_{0} \Phi_{0}}+1\right)\left\{1-\left(\frac{l_{0}}{l}\right)^{c / \dot{u}}\right\}
$$

where, $l_{0}$ is the half length of an initial crack arising from a preexistent flaw. Especially for $(r / l) \gg$ $n \approx 2$, we can readily integrate eq 20 with eq 33 and 28 , that is, with the time dependent $\dot{u}(t)$, 


$$
\begin{aligned}
\int_{l_{0}}^{l} \frac{\mathrm{d} l}{l}= & \frac{\exp \left(B \sigma_{\mathrm{e}}\right)}{\exp (Q / k T)} \int_{0}^{t}\left\{\frac{1}{\eta_{0}}+\frac{j_{\infty}}{\tau^{\prime}} \exp \left(-\frac{t}{\tau^{\prime}}\right)\right. \\
& +\dot{\gamma}(t)\} \mathrm{d} t
\end{aligned}
$$

Here, assuming that

$$
\dot{\gamma}(t)=\gamma_{0}\left(\sigma_{0}\right) t^{z-1} \quad \text { with } z>1
$$

we obtain

$$
\begin{aligned}
\ln \left(\frac{l}{l_{0}}\right)= & \frac{\exp \left(B \sigma_{\Theta}\right)}{\exp (Q / k T)}\left\{\left(\gamma_{0} t^{z} / z\right)+\left(t / \eta_{0}\right)\right. \\
& \left.+j_{\infty}\left[1-\exp \left(-\frac{t}{\tau^{\prime}}\right)\right]\right\}
\end{aligned}
$$

which provides us with the $l(t)$ vs. $t$ relation of the initial behaviour of plastic deformation.

(ii) For the large crack, $l(t)>l_{1}$

Integrating eq 26 with

$\Lambda(t)=\phi \theta^{2}=\lambda=$ constant, and $Y=$ variable as given by eq $40, i$.e.,

$$
\dot{u} \lambda \Phi \int_{t_{1}}^{t} \mathrm{~d} t=\int_{l_{1}}^{l} \frac{n+\lambda \Phi Y^{2}}{l Y^{2}} \mathrm{~d} l
$$

and using $\left(l_{1} / w\right) \ll 1$, we have

$$
\begin{aligned}
\dot{u} \lambda \Phi\left(t-t_{1}\right) \approx & \left\{n\left(\frac{\beta}{\alpha}\right)^{2}+\lambda \Phi\right\} \ln \left(\frac{l}{l_{1}}\right) \\
& -\frac{2 n \beta l^{\nu}}{\alpha^{2} \nu w^{\nu}}+\frac{n l^{2 \nu}}{2 \alpha^{2} \nu w^{2 \nu}} .
\end{aligned}
$$

When, $\lambda \Phi \ll n(\beta / \alpha)^{2} \approx 1 / 2$,

$t \approx \frac{n}{\dot{u} \lambda \Phi}\left\{\left(\frac{\beta}{\alpha}\right)^{2} \ln \left(\frac{l}{l_{1}}\right)-\frac{2 \beta l^{\nu}}{\alpha^{2} \nu w^{2}}+\frac{l^{2 \nu}}{2 \alpha^{2} \nu w^{2 \nu}}\right\}+t_{1}$

which is equivalent to the result obtained from eq 27 instead of eq 26.

\section{Time Dependence of $C O D$}

We can derive the following differential equation from eq 12 and 13 ,

$$
\frac{\mathrm{d} y(\Delta l, t)}{\mathrm{d} t}=\dot{u} y(\Delta l, t)
$$

and we obtain $y(\Delta l, t)$ by integrating eq 54 with constant $\dot{u}, i$. e.

$$
y(\Delta l, t)=y(\Delta l, 0) \exp (\dot{u} t)
$$

which is applicable for all $\Delta l$. Then we have

$$
y(0, t)=y(0,0) \exp (\dot{u} t)
$$

or

$$
a(t)=a_{0} \exp (\dot{u} t)
$$

where twice $a(t)$ is the separation of a crack at its starting point and time $t$ as shown in Figure 7, and $a_{0}$ is equal to $a(0)$. Therefore, the slope on the plot of $\ln \left(a / a_{0}\right)$ vs. $t$ will provide us the information on the dislocation velocity through $\dot{u}$.

\section{An Interpretation of the Empirical Equation of Crack-Extension}

By an expanded modification of eq 4, we now write $r(t)$ as

$$
r(t) \approx \frac{\pi^{2}}{8}\left(\frac{\sigma_{\mathrm{a}} \sqrt{l}}{\sigma_{\mathrm{Y}}}\right)^{2 m}=\frac{\pi^{2}}{8}\left(\frac{K}{\sigma_{\mathrm{Y}}}\right)^{2 m}
$$

with $K=\sigma_{\mathrm{a}} \sqrt{l}$ as the stress intensity factor. Since the rate of crack-extension can be plainly given by eq 27 for large $l(t)$, one has

$$
\frac{\mathrm{d} l}{\mathrm{~d} t}=\frac{\pi^{2} \dot{u} K^{2 m}}{8 n \sigma_{\mathrm{Y}}^{2 m}}
$$

with eq 58. Let's compare eq 1 with eq 59, and then we have the relation

$$
h=\left(\frac{\pi^{2} \dot{u}}{8 n}\right) / \sigma_{\overline{\mathrm{Y}}}{ }^{2 m}
$$

which provides a physical meaning to the proportional constant $h$ in eq 1 . If the empirical rule of Kitagawa, et al., ${ }^{38}$ for the fatigue crack-growth,

$$
h=p q^{2 m}
$$

were valid for our present case, we could rewrite eq 60 as

$$
h=\left\{\left(\frac{\pi^{2} \dot{u}}{8 n}\right)^{\ln q} \sigma_{\overline{\mathrm{Y}}}^{\ln p}\right\}^{1 / \ln \left(q \sigma_{\mathrm{X}}\right)}
$$

with positive constants $p$ and $q$.

\section{Dependence of Life Time on Applied Stress}

We obtain the following equation from eq 53 , 24,28 , and 35 by neglecting the correction term due to the finite width,

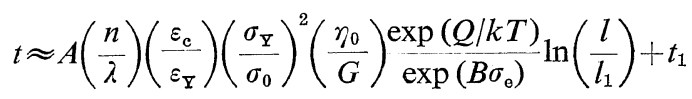

where, $A=(8 / \pi)(\beta / \alpha)^{2} \approx 2 / 3$. Furthermore from eq 63 , one has an approximate equation for the life time of $t=t_{\mathrm{b}}$ at a half crack length of $l=l_{\mathrm{b}}$ 
such as

$$
\begin{gathered}
t_{\mathrm{b}}=C\left(\sigma_{0} / \sigma_{\mathrm{Y}}\right)^{-f} \exp (Q / k T) \exp \left(-B \sigma_{\mathrm{e}}\right) \\
\approx C\left(\sigma_{0} / \sigma_{\mathrm{Y}}\right)^{-f} \exp (Q / k T) \\
\text { at low stress }\left(\sigma_{i} \leq \sigma_{0} \leq \sigma_{\mathrm{e}}\right) \\
\approx C \exp (Q / k T) \exp \left(-D \sigma_{0}\right) \\
\text { at high stress }\left(\sigma_{\mathrm{c}} \leq \sigma_{0} \ll \sigma_{\mathrm{Y}}\right)
\end{gathered}
$$

where, $f=2$ for the Dugdale model, and $C$ is given as

$$
C=\left\{A\left(\frac{n}{\lambda}\right)\left(\frac{\varepsilon_{\mathrm{c}}}{\varepsilon_{\mathrm{Y}}}\right) \ln \left(l_{\mathrm{b}} / l_{0}\right)\right\}\left(\frac{\eta_{0}}{G}\right)
$$

with $l_{0}$ in place of $l_{1}$ in eq 63 for simplicity. As is obvious from eq 64 and 66 , the relation

$$
\exp \left(D \sigma_{0}\right)=\left(\sigma_{0} / \sigma_{\bar{\Upsilon}}\right)^{f} \exp \left(B \sigma_{\Theta}\right)
$$

is valid for finite $\sigma_{\mathrm{e}}$. Thus, the material constant $D$ is given as

$$
D \approx B\left(1-\frac{\sigma_{i}}{\sigma_{0}}\right)+f\left(\frac{1}{\sigma_{\mathbf{Y}}}-\frac{1}{\sigma_{0}}\right)
$$

considering $\left(\sigma_{0} / \sigma_{\bar{Y}}\right)$ to be sufficiently small. In the case of constant stress, $B$ is equal to the stress sensitivity or $\left(v^{*} / k T\right)$ given in the eq 29 .

Approximate eq 65 and 66 imply that the applied stress dependence of life time changes markedly in the vicinity of a characteristic stress $\sigma_{\mathrm{c}}$. From the slope of eq 65 which equals that of eq 66 at $\sigma_{0}=\sigma_{\mathrm{c}}$, we have

$$
\sigma_{\mathrm{c}}=\frac{f}{D}
$$

which is reformed with eq 69 as

$$
\sigma_{\mathrm{c}}=\frac{2 f+B \sigma_{i}}{B+\left(f / \sigma_{\mathrm{Y}}\right)}
$$

where $B$ is nearly constant or decreases with the increasing temperature. $^{50}$ So eq 71 shows that $\left(\mathrm{d} \sigma_{\mathrm{c}} / \mathrm{d} T\right)<0$, observed by many workers, ${ }^{39}$ is due to negative terms of $\left(\mathrm{d} \sigma_{i} / \mathrm{d} T\right)$ and $\left(\mathrm{d} \sigma_{\bar{x}} / \mathrm{d} T\right)$.

The pre-exponential factor $C$ in eq $64-66$ is proportional to $\left(\eta_{0} / G\right)$ as shown in eq 67 , and has the dimension of time as seen from eq 35 . It should be noted that the eq 65 and 66 are nothing else but the so-called Holland-Turner's equation ${ }^{40}$ and the Zhurkov-Narsulaev's equation, ${ }^{41}$ respectively.

\section{CONCLUDING REMARKS}

In this work, we assumed the single rate process for the overall strain rate of plastic deformations and ignored the effect of anealing, recrystallization and other structural change. The formulated results thus need some rectifications in their application to cases of the structural change, and/or to temperature regions of multiple rate process. Nevertheless, eq $63-66$ provide us a clue for showing some material parameters affecting life time. For example, we can reckon the effect of molecular weight, $M$ through the term of $\eta_{0} \propto M^{\omega}$ with $\omega \approx 3.4$, the effect of a distribution of dislocation density through the parameter $n$, and a stress relaxation effect through the parameter $\lambda$, and that of yield stress, $\sigma_{\mathbf{Y}}$.

The effect of environmental reagents on crazing and cracking of stressed polymers have been reported by several authors as cavitation by stressaided solvation, ${ }^{42}$ a reduction of the critical strain of craze-initiation, ${ }^{43}$ a dilation by solvation, ${ }^{44}$ and a reduction of the cohesive energy which is controlled by the flow rate of liquids through the crack and craze. ${ }^{2,45-47}$ The present author also takes an interest in a mechanism of the environmental stress cracking of polymers, and has made an effort to find how to improve the life time of stressed polymers. The effect of the reagents remains unknown in this work since we did not explicitly consider the environmental effects in our model. However, from eq 27, 28, and 63, we can speculate as follows; the environmental stress may enhance predominantly the plastic deformation rate through the increase of the activation volume as well as a reduction of the true activation energy. The activation volume of polycarbonate with kerosene is actually larger than that without kerosene over a wide range of temperature, although the true activation energy does not change in this case. ${ }^{48}$

A part of this paper was presented at the 14 th Symposium on "Strength, Fracture and Fatigue of Materials" at Kyoto, July 21-22, 1977, held by the Society of Materials Science, Japan.

Acknowledgment. The author is gratefull to Dr. M. Kitagawa of Kanazawa University and to Dr. T. Hata of Gunma University for their critical comments and for providing very instructive literature. 


\section{Stress-Crack Extension in Polymeric Materials}

\section{APPENDIX}

\section{List of Symbols}

$a(t) \quad$, COD of crack-starting point at time $t$

$a_{0} \quad, a(t)$ at initial state $=a(0)$

$A$, parameter $=(8 / \pi)(\beta / \alpha)^{2}$

$b^{*} \quad$, stress sensitivity $=\left(\partial \ln \dot{u} / \partial \sigma_{\theta}\right)$

$B \quad$, material constant $=b^{*} \theta=\left(v^{*} \theta / k T\right)$

$c$, parameter related to relaxation time $=2 / \tau$

$C \quad$, material constant $=A(n / \lambda)\left(\varepsilon_{\mathrm{c}} / \varepsilon_{\mathrm{Y}}\right)\left(\eta_{0} / G\right) \ln \left(l_{\mathrm{b}} / l_{0}\right)$

COD , crack-opening displacement

$D \quad$ material constant $\approx B\left[1-\left(\sigma_{i} / \sigma_{0}\right)\right]+f\left[\sigma_{\mathrm{Y}}{ }^{-1}-\sigma_{0}{ }^{-1}\right]$

$f \quad$, parameter as to applied stress dependence of life time $=2$ for the Dugdale model

$G \quad$, material constant as to the elastic modulus $=(1+\rho)$

$h$, proportional constant of $i$ to $K^{2 m}$ in eq 1

$J(t)$, creep function at time $t$

$j_{0} \quad$, instantaneous creep compliance

$j_{\infty}$, creep compliance at infinite time

$k$, Boltzmann's constant

$K$, stress intencity factor

$l, l(t)$, half crack-length at time $t$

$l^{*}(t), l(t)$ of imaginary crack

$l_{\mathrm{b}} \quad$, half crack-length at time $t_{\mathrm{b}}$

$l_{0} \quad$, half crack-length of preexisted flaw

$l_{1} \quad$, lower limit of $l$ recognizable by eye

$i$, rate of crack-extension

$m$, exponent of $K$ in eq 1

$M \quad$, molecular weight

$n$, shape parameter for the distribution of plastic strain or dislocation density in plastic zone

$p \quad$, parameter related to $h$ as shown in eq 61

$q$, parameter related to $h$ as shown in eq 61

$Q \quad$, true activation energy for overall strain rate $\dot{u}$

$Q_{\text {a }}$, apparent activation energy for $\dot{u}$

$r, r(t)$, length of plastic zone at time $t$

$s \quad$, stress sensitivity $=b^{*}$

$t \quad$, time

$t_{\mathrm{b}} \quad$, time to failure

$t_{\infty}$, infinite time

$T$, temperature

$u$, overall strain of plastic deformation

$\dot{u}$, overall strain rate of plastic deformation

$\dot{u}_{0} \quad$, rate constant related to $\dot{u}$ as shown in eq 28

$v^{*}$, shear activation volume

$w \quad$, half width of specimen

$x$, distance from crack-tip

$y(x, t)$, half thickness of plastic zone at time $t$ and distance $x$

$y^{*}(x, t), y(x, t)$ due to imaginary crack

$Y \quad$, finite width correction factor
$Y_{0} \quad$, constant as to $Y$ at $l=0$

$z$, exponent of $t$ for $\dot{\gamma}(t)$ as shown in eq 49

$\alpha \quad$, parameter for $Y$ in this work

$\beta$, parameter for $Y$ in this work

$\dot{\gamma}(t)$, strain rate component due to growth of voids

$\gamma_{0}$, rate constant for $\dot{\gamma}(t)$

$\delta \quad$, constant $=-[\mathrm{d} \ln (r / l) / \mathrm{d} \ln (l / w)]$

$\varepsilon_{\mathrm{c}}$, critical strain of craze-initiation

$\varepsilon_{\mathrm{Y}} \quad$, yield strain

$\zeta$, parameter, unity for the Dugdale model, and $\left(\varepsilon_{\mathrm{Y}} / \varepsilon_{\mathrm{c}}\right) \phi(t)$ for the Kitagawa-Motomura model

$\eta_{0}$, Newton viscosity

$\theta$, degree of stress relaxation at equillibrium state $=\Theta\left(t_{\infty}\right) \approx 0.2 \sim 0.4$

$\Theta(t)$, time function of stress relaxation, $\sigma(t) / \sigma_{0} \approx \exp (-t / \tau)$

$\lambda \quad, \Lambda(t)$ at equillibrium state $=\Lambda\left(t_{\infty}\right)$

$\Lambda(t)$, time function $=\Theta^{2}(t) \phi(t)$

$\nu$, parameter for $Y$ in this work

$\xi \quad$, function $=[1-(x / r)]^{1 / 2}$

$\rho$, material constant related to elastic modulus

$\sigma_{\mathrm{a}}(t)$, applied stress uncorrected at time $t$

$\sigma_{\mathrm{Y}}$, yield stress

$\sigma(t) \quad$, corrected stress $=\sigma_{0} \Theta(t) Y / \sqrt{\pi}$ at time $t$

$\sigma_{i} \quad$, internal stress, level of $\sigma_{i}$ is given as $\sigma(t)$ at $[\mathrm{d} \sigma(t) / \mathrm{d} \ln t]=0$ after $\mathrm{Li}^{4}{ }^{4}$, viz., $\theta \sigma_{0}$ or $\sigma\left(t_{\infty}\right)$.

$\sigma_{0} \quad$, initial stress applied $=\sigma(0)$

$\sigma_{\mathrm{e}} \quad$, effective stress $=\sigma(t)-\sigma_{i}$, or $\sigma_{0}-\sigma_{i}$ for constant $\sigma_{0}$

$\sigma_{\mathrm{c}}$, characteristic stress given by eq 71

$\tau$, relaxation time

$\tau^{\prime}$, retardation time

$\Phi \quad$, material constant $=(\pi / 8)\left(\varepsilon_{\mathrm{Y}} / \varepsilon_{\mathrm{c}}\right)\left(\sigma_{0} / \sigma_{\mathrm{Y}}\right)^{2}$

$\Phi_{0} \quad$, material constant $=Y_{0}^{2} \Phi$

$\phi(t)$, creep compliance of the Kitagawa-Motomura model

$\phi_{0} \quad, \phi(t)$ at initial state $=\phi(0)$

$\psi \quad$, constant for $\phi(t)$ at $t \neq 0$

$\psi^{*}, \phi^{*}(t)$, creep compliance in this work

$\dot{\phi}^{*}(t)$, one component of $\dot{u}_{0}$ due to $\left(\mathrm{d} \psi^{*} / \mathrm{d} t\right)$

$\omega$, exponent in relation of $\eta_{0} \propto M^{\omega}$

$\Delta \quad$, symbol of increment

\section{REFERENCES}

1. Topics on ESC of polymeric materials was pertinently reviewed by P. D. Calvert, Nature, 265, Jan. 13, 104 (1977).

2. G. P. Marshall, et al., Proc. R. Soc. London, Ser. A, 319, 165 (1970).

3. G. P. Marshall, et al., Soc. Plast. Eng. J., 28, Sept., 26 (1972).

4. J. G. Williams, et al., Pure and Appl. Chem., 39, 275 (1974). 
5. J. G. Williams, Polym. Eng. Sci., 17, 144 (1977).

6. P. Paris and F. Erdogan, Trans. ASME, Ser. D, 85, 528 (1963).

7. M. L. Williams, in "Fracture of Solids," D. C. Drucker and J. J. Gilman Ed., Gordon and Breach Science Publishers, New York, N. Y., 1963, p 157.

8. W. G. Knauss, in "Deformation and Fracture of High Polymers," H.H. Kausch, et al., Ed., Plenum Press, New York, N. Y., 1973, p 501.

9. R. J. Ferguson, et al., Polymer, 14, 451 (1973).

10. D. S. Dugdale, J. Mech. Phys. Solids, 8, 100 (1960).

11. H. R. Brown and I. M. Ward, Polymer, 14, 469 (1973).

12. N.J. Mills and N. Walker, Polymer, 17, 335 (1976).

13. J. N. Goodier and F. A. Field, (1963), or F. M. Burdekin and D. E. Stone, (1966) quoted by R. D. Hoffman and O. Richmond, J. Appl. Phys., 47, 4289 (1976).

14. G. P. Morgan and I. M. Ward, Polymer, 18, 87 (1977).

15. For example, P. Beahan, et al., Polymer, 14, 96 (1973).

16. R. P. Kambour, Appl. Polym. Symp., 7, 215 (1968).

17. D. Hull in "Deformation and Fracture of High Polymers," H. H. Kausch, et al., Ed., Plenum Press, New York, N. Y., 1973, p 171.

18. E. H. Andrews, "The Physics of Glassy Polymers," R. N. Haward, Ed., Applied Science Publishers Ltd., London, 1973, p 423.

19. T. W. Owen and D. Hull, Polymer, 14, 476 (1973).

20. K. Shigematsu, et al., J. Polym. Sci., Polym. Phys. Ed., 13, 73 (1975).

21. C. J. Farrel and A. Keller, J. Mater. Sci., 12, 966 (1977).

22. A. G. Kolbeck and D. R. Uhlmann, J. Polym. Sci., Polym. Phys. Ed., 14, 1257 (1976), and references cited in it.

23. H. Gleiter, et al., "Deformation and Fracture of High Polymers," H.H. Kausch, et al., Ed., Plenum Press, New York, N. Y., 1973, p 149.

24. For example, F. R. N. Nabarro, "Theory of Crystal Dislocations," Oxford University Press, London, 1967, p 9, p 40 and p 67.

25. H. Riedel, Mater. Sci. Eng., 30, 187 (1977).

26. J. R. Rice, (1966, or 1968) quoted by G.P. Morgan, et al., in Polymer, 18, 87 (1977), or by N. J. Mills, et al., in Polymer, 17, 335 (1976).

27. M. Kitagawa and K. Motomura, J. Polym. Sci., Polym. Phys. Ed., 12, 1979 (1974).

28. W. F. Brown Jr. and J. E. Srawley, Am. Soc. Test. Mater. Spec. Tech. Publ., No. 410, pp 1-145, (1966), especially pp 12-13 for the tension and bending mode.

29. "Environmental Stress-Cracking of Ethylene Plastics," Am. Soc. Test. Mater. D1693-70.

30. A. H. Cottrell, "Dislocations and Plastic Flow in Crystals," Clarendon Press, Oxford, 1953, pp 1718.

31. R. D. Nicholson, Mater. Sci. Eng., 22, 1 (1976).

32. For example, W. Wu and A. P. L. Turner, $J$. Polym Sci., Polym. Phys. Ed., 13, 19 (1975); or J. Kubat and M. Rigdahl, Mater. Sci. Eng., 24, 223 (1976).

33. A. S. Krausz and H. Eyring, "Deformation Kinetics," John Wiley \& Sons, New York, N. Y., 1975, p 96.

34. R. N. Haward and G. Thackray, Proc. R. Soc. London, Ser. A, 302, 453 (1968); or P. B. Bowden in "The Physics of Glassy Polymers," R. N. Haward Ed., Applied Science Publishers Ltd., London, 1973, p. 298.

35. J. C.M.Li, et al., in "Deformation and Fracture of High Polymers," H. H. Kausch, et al., Ed., Plenum Press, New York, N. Y., 1973, pp 241-242.

36. A. S. Krausz and H. Eyring in "Deformation Kinetics," John Wiley \& Sons, New York, N. Y., 1975, p 107 and p 117.

37. J. Kubát, et al., J. Appl. Polym. Sci., 20, 2799 (1976).

38. H. Kitagawa and M. Misumi, "Preprints of the Japan Society of Mechanical Engineering," No. 714-10, 67 (1971).

39. For example, Hostalen GM 5010 pressure pipe, in Hoechst Plastics, Aug., 15 (1971).

40. A. J. Holland and W. E. S. Turner, J. Soc. Glass Tech., 24, 46 (1940); or Chem. Abst., 34, 4533-9 (1940).

41. S. N. Zhurkov and B. N. Narsulaev, J. Tech. Phys. USSR, 23, 1677 (1953); or S. N. Zhurkov, Int. J. Fract. Mech., 1, 311 (1965).

42. E. H. Andrews and L. Bevan, Polymer, 13, 337 (1972).

43. G. A. Bernier and R. P. Kambour, Macromolecules, 1, 145 (1968).

44. A. N. Gent, J. Mater. Sci., 5, 925 (1970).

45. J. G. Williams and G.P. Marshall in "Deformation and Fracture of High Polymers," H. H. Kausch, et al., Ed., Plenum Press, New York, N. Y., 1973, pp 557-583.

46. K. Matsushige, et al., J. Macromol. Sci., B11, 565 (1975).

47. M.E.R. Shanahan and J. Shultz, J. Polym. Sci., Polym. Phys. Ed., 14, 1567 (1976).

48. I. Narisawa and T. Kondo, J. Soc. Mater. Sci. Jpn., 21, 321 (1972).

49. J. C. M. Li, Can. J. Phys., 45, 493 (1967).

50. A. S. Krausz, Mater. Sci. Eng., 6, 260 (1970). 